\title{
The Error Norm of Certain Gaussian Quadrature Formulae
}

\author{
By G. Akrivis*
}

\begin{abstract}
We consider Gauss quadrature formulae $Q_{n}, n \in \mathbf{N}$, approximating the integral $I(f):=\int_{-1}^{1} w(x) f(x) d x, w=W / p_{i}, i=1,2$, with $W(x)=(1-x)^{\alpha}(1+x)^{\beta}, \alpha, \beta= \pm 1 / 2$ and $p_{1}(x)=1+a^{2}+2 a x, p_{2}(x)=(2 b+1) x^{2}+b^{2}, b>0$. In certain spaces of analytic functions the error functional $R_{n}:=I-Q_{n}$ is continuous. In [1] and [2] estimates for $\left\|R_{n}\right\|$ are given for a wide class of weight functions. Here, for a restricted class of weight functions, we calculate the norm of $R_{n}$ explicitly.
\end{abstract}

1. Introduction. Consider the integral $I$,

$$
I(f)=\int_{-1}^{1} w(x) f(x) d x, \quad w \geqslant 0,\|w\|_{1}>0,
$$

approximated by the Gaussian quadrature formula $Q_{n}$,

$$
Q_{n}(f)=\sum_{i=1}^{n} w_{i} f\left(x_{i}\right) \text {. }
$$

Let $P_{k}, P_{k}(x)=\alpha_{k} x^{k}+\beta_{k} x^{k-1}+\cdots, \alpha_{k}>0, k \in \mathbf{N}_{0}$, be the orthonormal polynomials corresponding to the weight function $w$, i.e.,

$$
\int_{-1}^{1} w(x) P_{i}(x) P_{j}(x) d x=\delta_{i j} .
$$

The following classical representation for the error term $R_{n}(f):=I(f)-Q_{n}(f)$ can be found, e.g., in [4, p. 75],

$$
\bigwedge_{f \in C^{2 n}[-1,1]} \bigvee_{\xi \in(-1,1)} R_{n}(f)=\frac{1}{(2 n) ! \alpha_{n}^{2}} f^{(2 n)}(\xi) .
$$

The estimate

$$
\left|R_{n}(f)\right| \leqslant \frac{1}{(2 n) ! \alpha_{n}^{2}}\left\|f^{(2 n)}\right\|_{\infty}
$$

following immediately from (1.1), is often unsatisfactory, since bounds for higher derivatives are required, and, in addition, the calculation usually has to be repeated for different values of $n$.

For analytic functions Hämmerlin [8] suggested the following method for obtaining derivative-free error estimates: Let $q_{\kappa}(x):=x^{\kappa}, \kappa \in \mathbf{N}_{0}, r>1$ and $C_{r}:=\{z \in \mathbf{C}$ : $|z|<r\}$. For a function $f$ holomorphic in $C_{r}$,

$$
f(z)=\sum_{\kappa=0}^{\infty} \alpha_{\kappa}^{f} z^{\kappa}, \quad z \in C_{r},
$$

Received August 6, 1984; revised December 5, 1984.

1980 Mathematics Subject Classification. Primary 65D30.

*Current address: Department of Mathematics, University of Crete, Iraklion, Crete, Greece. 
define

$$
|f|_{r}:=\sup \left\{\left|\boldsymbol{\alpha}_{\kappa}^{f}\right| r^{\kappa}: \kappa \in \mathbf{N}_{0} \text { and } R_{n}\left(q_{\kappa}\right) \neq 0\right\}
$$

In the space

$$
X_{r}:=\left\{f: f \text { holomorphic in } C_{r} \text { and }|f|_{r}<\infty\right\}
$$

$|\cdot|_{r}$ is a seminorm. The error functional $R_{n}$ is continuous in $\left(X_{r},|\cdot|_{r}\right)$, and for the error norm

$$
\left\|R_{n}\right\|:=\sup \left\{\frac{\left|R_{n}(f)\right|}{|f|_{r}}: f \in X_{r},|f|_{r} \neq 0\right\}
$$

the relation

$$
\left\|R_{n}\right\|=\sum_{\kappa=0}^{\infty} \frac{\left|R_{n}\left(q_{\kappa}\right)\right|}{r^{\kappa}}
$$

holds (see [8], [1], [2]).

For the weight functions considered here, either the condition

$$
w(\cdot) / w(-\cdot) \text { is nondecreasing }
$$

or the condition

$$
w(\cdot) / w(-\cdot) \text { is nonincreasing }
$$

is valid.

Condition (1.6) implies

$$
R_{n}\left(q_{\kappa}\right) \geqslant 0, \quad \kappa \in \mathbf{N}_{0}
$$

(see [5]). Thus, from (1.5) there follows

$$
\left\|R_{n}\right\|=\sum_{\kappa=0}^{\infty} \frac{R_{n}\left(q_{\kappa}\right)}{r^{\kappa}}=R_{n}\left(\sum_{\kappa=0}^{\infty} \frac{q_{\kappa}}{r^{\kappa}}\right)
$$

i.e.,

$$
\left\|R_{n}\right\|=r R_{n}(\varphi) \text { with } \varphi(x):=1 /(r-x) .
$$

Let the polynomial $\pi_{n-1}$ of degree less than $n$ interpolate the function $\varphi$ at the abscissae $x_{1}, \ldots, x_{n}$ of $Q_{n}$. Since $Q_{n}$ integrates $\pi_{n-1}$ exactly, $R_{n}(\varphi)=R_{n}\left(\varphi-\pi_{n-1}\right)$ holds. Setting $\Pi_{n}(x):=\left(x-x_{1}\right) \cdots\left(x-x_{n}\right)$, we obtain

$$
\varphi(x)-\pi_{n-1}(x)=\gamma_{n} \Pi_{n}(x) /(r-x),
$$

where $\gamma_{n}$ is a constant, because the function on the left-hand side vanishes at $x_{1}, \ldots, x_{n}$. Multiplying by $r-x$ and taking the limit as $x \rightarrow r$ we obtain $\gamma_{n}=$ $1 / \Pi_{n}(r)$ (see [3, pp. 71-72]). Thus, from (1.9) we get the representation

$$
\left\|R_{n}\right\|=\frac{r}{\Pi_{n}(r)} \int_{-1}^{1} w(x) \frac{\Pi_{n}(x)}{r-x} d x \quad \text { with } \Pi_{n}(x)=\prod_{i=1}^{n}\left(x-x_{i}\right),
$$

for weight functions satisfying (1.6).

If $w$ satisfies (1.7),

$$
(-1)^{\kappa} R_{n}\left(q_{\kappa}\right) \geqslant 0
$$

holds (see [5]), and we obtain similarly

$$
\left\|R_{n}\right\|=r R_{n}(\psi), \quad \psi(x):=1 /(r+x),
$$


and

$$
\left\|R_{n}\right\|=\frac{r}{\Pi_{n}(-r)} \int_{-1}^{1} w(x) \frac{\Pi_{n}(x)}{r+x} d x \quad \text { with } \Pi_{n}(x)=\prod_{i=1}^{n}\left(x-x_{i}\right) .
$$

In [1] and [2], estimates for $\left\|R_{n}\right\|$ were derived for weight functions satisfying (1.6) or (1.7), and $\left\|R_{n}\right\|$ was given for $w=W$. Starting from (1.10) or (1.13) respectively, in the next section we calculate the norm of $R_{n}$ for weight functions $w$ with

$$
\begin{aligned}
& w=W / p_{i}, \quad i=1,2, \\
& W(x)=(1-x)^{\alpha}(1+x)^{\beta}, \quad \alpha, \beta= \pm 1 / 2, \\
& p_{1}(x)=1+a^{2}+2 a x, \\
& p_{2}(x)=(2 b+1) x^{2}+b^{2}, \quad b>0 .
\end{aligned}
$$

Two numerical examples conclude the paper.

Remark. For even weight functions, (1.4) can be written as $|f|_{r}=\sup _{\kappa \geqslant n}\left\{\left|\alpha_{2 \kappa}^{f}\right| r^{2 \kappa}\right\}$ (cf. [1]). If $w(\cdot) / w(-\cdot)$ is strictly monotonic, then $R_{n}\left(q_{\kappa}\right) \neq 0$ for $\kappa \geqslant 2 n$ (see [5]), and $|\cdot|_{r}$ can be equivalently defined by $|f|_{r}:=\sup _{\kappa \geqslant 2 n}\left\{\left|\alpha_{\kappa}^{f}\right| r^{\kappa}\right\}$.

\section{The Norm of the Error Functional.}

a. $p_{1}(x)=1+a^{2}+2 a x$. The case $a=0, \pm 1$ is treated in [1], [2] if $w$ remains integrable. For $|a|<1, a \neq 0$, put $d:=1 / a$ to obtain $p_{1}(x)=a^{2}\left(1+d^{2}+2 d x\right)$, $|d|>1$. Therefore we only consider the case $|a|>1$.

We first summarize some results of Kumar [9] which are important for the subsequent development.

LEMMA 1. Let $p_{1}(x)=1+a^{2}+2 a x,|a|>1 ; W(x)=(1-x)^{\alpha}(1+x)^{\beta}$ and $w=W / p_{1}$. Let $T_{i}$ and $U_{i}$ be the Chebyshev polynomials of the first and second kind, respectively. Then the abscissae $x_{1}, \ldots, x_{n}$ of the Gauss quadrature formula $Q_{n}$ corresponding to $w$ are the zeros of

(i) $a T_{n}+T_{n-1}$ if $\alpha=\beta=-1 / 2$,

(ii) $a U_{n}+U_{n-1}$ if $\alpha=\beta=1 / 2$,

(iii) $a U_{n}+(1+a) U_{n-1}+U_{n-2}$ if $\alpha=-\beta=1 / 2$ and $n>1$.

Remark. For $\alpha=\beta= \pm 1 / 2$ the condition (1.6) is satisfied if $a<-1$, the condition (1.7) if $a>1$. For $\alpha=-\beta=-1 / 2$, (1.6) holds, for $\alpha=-\beta=1 / 2$ we have (1.7).

We now establish the first of our results.

THEOREM 1. Consider $p_{1}(x)=1+a^{2}+2 a x,|a|>1, W(x)=(1-x)^{\alpha}(1+x)^{\beta}$, $w=W / p_{1}$. Let $\tau:=r-\sqrt{r^{2}-1}$. For the norm of the error functional $R_{n}$ the following is true:

$$
\left\|R_{n}\right\|=\frac{2 \pi r \tau^{2 n}}{(\tau+a)\left[\tau\left(1+\tau^{2 n-2}\right)+a\left(1+\tau^{2 n}\right)\right] \sqrt{r^{2}-1}}
$$

for $\alpha=\beta=-1 / 2$ and $a<-1$,

$$
\left\|R_{n}\right\|=\frac{2 \pi r \tau^{2 n+2} \sqrt{r^{2}-1}}{(\tau+a)\left[\tau\left(1-\tau^{2 n}\right)+a\left(1-\tau^{2 n+2}\right)\right]}
$$


for $\alpha=\beta=1 / 2$ and $a<-1$,

$$
\left\|R_{n}\right\|=\frac{2 \pi r \tau^{2 n+1}}{(\tau-a)\left[\tau\left(1+\tau^{2 n-1}\right)-a\left(1+\tau^{2 n+1}\right)\right]}\left(\frac{r+1}{r-1}\right)^{1 / 2}
$$

for $\alpha=-\beta=1 / 2$ and $n>1$.

Proof. First, let us verify the identity (2.1). The weight function $w$ satisfies condition (1.6) for $\alpha=\beta=-1 / 2$ and $a<-1$. Thus, by Lemma 1 (i) and (1.10),

$$
\left\|R_{n}\right\|=\frac{r}{a T_{n}(r)+T_{n-1}(r)} \int_{-1}^{1}\left(1-x^{2}\right)^{-1 / 2} \frac{a T_{n}(x)+T_{n-1}(x)}{(r-x)\left(1+a^{2}+2 a x\right)} d x
$$

holds. Let the integral on the right-hand side of (2.4) be denoted by $I_{n}(a, r)$. Substituting $x=\cos y$ we obtain

$$
I_{n}(a, r)=\int_{0}^{\pi} \frac{a \cos (n y)+\cos [(n-1) y]}{(r-\cos y)\left(1+a^{2}+2 a \cos y\right)} d y .
$$

Set

$$
C_{n}(a):=2 a \int_{0}^{\pi} \frac{a \cos (n y)+\cos [(n-1) y]}{1+a^{2}+2 a \cos y} d y
$$

to obtain

$$
I_{n}(a, r)=\frac{1}{1+a^{2}+2 a r}\left\{\int_{0}^{\pi} \frac{a \cos (n y)+\cos [(n-1) y]}{r-\cos y} d y+C_{n}(a)\right\} .
$$

Since

$$
\int_{0}^{\pi} \frac{\cos (m y)}{r-\cos y} d y=\frac{\pi \tau^{m}}{\sqrt{r^{2}-1}}
$$

(cf., e.g., [7, p. 112]), we have

$$
I_{n}(a, r)=\frac{1}{1+a^{2}+2 a r}\left\{\frac{\pi \tau^{n-1}(a \tau+1)}{\sqrt{r^{2}-1}}+C_{n}(a)\right\} .
$$

By (1.5), $\left\|R_{n}\right\|=O\left(r^{-2 n}\right)$ holds for $r \rightarrow \infty$, and (2.4) yields $I_{n}(a, r)=O\left(r^{-n-1}\right)$ for $r \rightarrow \infty$. Therefore $C_{n}(a)=0$, which can also be established by straightforward calculation. Thus,

$$
I_{n}(a, r)=\frac{\pi \tau^{n}}{(\tau+a) \sqrt{r^{2}-1}} .
$$

Combining this with $T_{m}(r)=\left[\left(r-\sqrt{r^{2}-1}\right)^{m}+\left(r+\sqrt{r^{2}-1}\right)^{m}\right] / 2$ (see [11, p. 5]), the relation (2.1) follows from (2.4).

(2.2) can be proved in a similar way. To prove (2.3), use the relation

$$
(1-x)\left[U_{m}(x)+U_{m-1}(x)\right]=T_{m}(x)-T_{m+1}(x)
$$

which immediately follows from well-known identities for Chebyshev polynomials (cf., e.g., [11, p. 9]).

Remark. $I_{n}(a, r)$ is also calculated by Kumar [9] by means of the generating function for the polynomials $a T_{n}+T_{n-1}$. 
COROllary 1. Let $p_{1}(x)=1+a^{2}+2 a x,|a|>1, W(x)=(1-x)^{\alpha}(1+x)^{\beta}$ and $w=W / p_{1}$. Then the norm of $R_{n}$ can be expressed as

$$
\left\|R_{n}\right\|=\frac{2 \pi r \tau^{2 n}}{(\tau-a)\left[\tau\left(1+\tau^{2 n-2}\right)-a\left(1+\tau^{2 n}\right)\right] \sqrt{r^{2}-1}}
$$

if $\alpha=\beta=-1 / 2$ and $a>1$, and as

$$
\left\|R_{n}\right\|=\frac{2 \pi r \tau^{2 n+2} \sqrt{r^{2}-1}}{(\tau-a)\left[\tau\left(1-\tau^{2 n}\right)-a\left(1-\tau^{2 n+2}\right)\right]}
$$

if $\alpha=\beta=1 / 2$ and $a>1$, and as

$$
\left\|R_{n}\right\|=\frac{2 \pi r \tau^{2 n+1}}{(\tau+a)\left[\tau\left(1+\tau^{2 n-1}\right)+a\left(1+\tau^{2 n+1}\right)\right]}\left(\frac{r+1}{r-1}\right)^{1 / 2}
$$

if $\alpha=-\beta=-1 / 2$ and $n>1$.

Proof. Let $R_{n}$ and $R_{n}^{*}$ be the error functionals corresponding to the weight functions $w$ and $w(-\cdot)$, respectively. Then obviously $R_{n}\left(q_{\kappa}\right)=(-1)^{\kappa} R_{n}^{*}\left(q_{\kappa}\right)$ holds, and thus $\left\|R_{n}\right\|=\left\|R_{n}^{*}\right\|$. Hence, the corollary immediately follows from Theorem 1 .

b. $p_{2}(x)=(2 b+1) x^{2}+b^{2}, b>0$. We first summarize some results of Kumar [10] which are neetled in the sequel.

LEMMA 2. Let $p_{2}(x)=(2 b+1) x^{2}+b^{2}, b>0, W(x)=(1-x)^{\alpha}(1+x)^{\beta}$ and $w=W / p_{2}$. The abscissae $x_{1}, \ldots, x_{n}$ of the Gauss quadrature formula $Q_{n}$ corresponding to $w$ are the zeros of

(i) $(2 b+1) T_{n}+T_{n-2}$ if $\alpha=\beta=-1 / 2$ and $n>1$,

(ii) $(2 b+1) U_{n}+U_{n-2}$ if $\alpha=\beta=1 / 2$ and $n>1$,

(iii) $(2 b+1)\left(U_{n}+U_{n-1}\right)+U_{n-2}+U_{n-3}$ if $\alpha=-\beta=1 / 2$ and $n>2$.

Our second result is presented in the following theorem.

THEOREM 2. Let $p_{2}(x)=(2 b+1) x^{2}+b^{2}, b>0, W(x)=(1-x)^{\alpha}(1+x)^{\beta}$ and $w=W / p_{2}$. For the norm of the error functional we have:

$$
\left\|R_{n}\right\|=\frac{4 \pi r \tau^{2 n}}{(b+r \tau)\left[(2 b+1)\left(1+\tau^{2 n}\right)+\tau^{2}\left(1+\tau^{2 n-4}\right)\right] \sqrt{r^{2}-1}}
$$

for $\alpha=\beta=-1 / 2, n>1$,

$$
\left\|R_{n}\right\|=\frac{4 \pi r \tau^{2 n+2} \sqrt{r^{2}-1}}{(b+r \tau)\left[(2 b+1)\left(1-\tau^{2 n+2}\right)+\tau^{2}\left(1-\tau^{2 n-2}\right)\right]}
$$

for $\alpha=\beta=1 / 2, n>1$, and

$$
\left\|R_{n}\right\|=\frac{4 \pi r \tau^{2 n+1}}{(b+r \tau)\left[(2 b+1)\left(1+\tau^{2 n+1}\right)+\tau^{2}\left(1+\tau^{2 n-1}\right)\right]}\left(\frac{r+1}{r-1}\right)^{1 / 2}
$$

for $\alpha=-\beta=1 / 2, n>2$.

Proof. In this case (1.7) holds, and the results follow from (1.13) using Lemma 2.

Symmetry arguments yield the following corollary.

Corollary 2. Let $w(x)=((1+x) /(1-x))^{1 / 2} /\left[(2 b+1) x^{2}+b^{2}\right], b>0$. The norm of the error functional corresponding to $w$ is then given by (2.10) also. 
Remark. Let $K_{n}(z):=R_{n}\left(\varphi_{z}\right), \varphi_{z}(x):=1 /(z-x),|z|=r$. If $f$ is holomorphic in a region $B$ including $C_{r}$ the representation

$$
R_{n}(f)=\frac{1}{2 \pi i} \int_{C_{r}} K_{n}(z) f(z) d z
$$

holds. Gautschi and Varga [6] showed that for weight functions satisfying either (1.6) or (1.7)

$$
\max _{|z|=r}\left|K_{n}(z)\right|=\max \left\{K_{n}(r),\left|K_{n}(-r)\right|\right\}=\sum_{\kappa=0}^{\infty} \frac{\left|R_{n}\left(q_{\kappa}\right)\right|}{r^{\kappa+1}}
$$

holds. Therefore, we have $\max _{|z|=r}\left|K_{n}(z)\right|=\left\|R_{n}\right\| / r$, and for the weight functions considered here $\max _{|z|=r}\left|K_{n}(z)\right|$ has also been determined.

3. Numerical Results. For $f \in X_{\rho},\left|R_{n}(f)\right|$ is bounded by $\left\|R_{n}\right\||f|_{r}, r \in(1, \rho]$. Therefore,

$$
\left|R_{n}(f)\right| \leqslant \inf _{1<r \leqslant \rho}\left(\left\|R_{n}\right\||f|_{r}\right)
$$

holds. (Although not explicitly noted, $\left\|R_{n}\right\|$ is obviously a function of $r$.) Estimating $|f|_{r}$ by $\|f\|_{2, r}$,

$$
\|f\|_{2, r}:=\frac{1}{\sqrt{2 \pi r}}\left(\int_{|z|=r}|f(z)|^{2}|d z|\right)^{1 / 2},
$$

or by $\max _{|z|=r}|f(z)|$, which exist at least for $r<\rho$, we obtain

$$
\left|R_{n}(f)\right| \leqslant \inf _{1<r<\rho}\left(\left\|R_{n}\right\|\|f\|_{2, r}\right)
$$

and

$$
\left|R_{n}(f)\right| \leqslant \inf _{1<r<\rho}\left(\left\|R_{n}\right\| \max _{|z|=r}|f(z)|\right)
$$

respectively (see [8]). The sharpness of these estimates is demonstrated by two numerical examples.

Example 1. Let $f(z):=\exp (z), f \in X_{r}, r>1(\rho=\infty)$. Approximate the integral

$$
\int_{-1}^{1} \frac{1}{(3+2 \sqrt{2})\left(1+x^{2}\right) \sqrt{1-x^{2}}} f(x) d x
$$

by the Gaussian quadrature formula $Q_{2}$ corresponding to

$$
w(x)=\frac{1}{(3+2 \sqrt{2})\left(1+x^{2}\right) \sqrt{1-x^{2}}} .
$$

The abscissae and the weights of $Q_{2}$ are given in [10]. The remainder term is $R_{2}(f)=2.016 \cdot 10^{-3}$. Setting $b=1+\sqrt{2}$ and $n=2$ in (2.8), we obtain the norm of the error functional $R_{2}$. With $|f|_{r}=r^{4} / 24$ for $1<r \leqslant \sqrt{30},|f|_{r}=r^{6} / 720$ for $\sqrt{30}<r \leqslant \sqrt{56}$, and so on, and $\max _{|z|=r}|f(z)|=\exp (r)$, (3.1) and (3.3) yield for $\left|R_{2}(f)\right|$ the bounds $2.019 \cdot 10^{-3}(r=5.45)$ and $1.073 \cdot 10^{-2}(r=4.15)$, respectively. 
Example 2. Let

$$
f(z)=\sum_{\kappa=4}^{\infty}\left(\frac{z}{2}\right)^{\kappa}=\frac{1}{8} \frac{z^{4}}{2-z}, \quad f \in X_{r} \text { for } r \in(1,2](\rho=2) .
$$

The remainder term $R_{2}(f)$ for the approximation of

$$
\int_{-1}^{1} \frac{1}{(5+4 x) \sqrt{1-x^{2}}} f(x) d x
$$

by the Gaussian quadrature formula $Q_{2}$ corresponding to

$$
w(x)=\frac{1}{(5+4 x) \sqrt{1-x^{2}}}
$$

is $7.18 \cdot 10^{-3}$. The abscissae of $Q_{2}$ are the zeros of $2 T_{2}+T_{1}$ (Lemma 1(i), $a=2$ ). We have

$$
|f|_{r}=\frac{r^{4}}{16}, \quad\|f\|_{2, r}=\left[\sum_{\kappa=4}^{\infty}\left(\frac{r}{2}\right)^{2 \kappa}\right]^{1 / 2}=\frac{r^{4}}{8 \sqrt{4-r^{2}}}
$$

(cf. [8]) and $\max _{|z|=r}|f(z)|=r^{4} /(16-8 r)$. Setting $a=2$ and $n=2$ in (2.5), we obtain the norm of $R_{2}$. Now, from (3.1), (3.2) and (3.3), we get for $\left|R_{2}(f)\right|$ the bounds $1.25 \cdot 10^{-2}(r=2), 3.06 \cdot 10^{-2}(r=1.65)$ and $8.75 \cdot 10^{-2}(r=1.50)$, respectively.

Acknowledgment. The author would like to thank Professor G. Hämmerlin for his encouragement during this work.

Mathematisches Institut der Universität

Theresienstrasse 39

D-8000 München 2, West Germany

1. G. Akrivis, "Fehlerabschätzungen für Gauss-Quadraturformeln,” Numer. Math., v. 44, 1984, pp. $261-278$.

2. G. Akrivis \& A. Burgstaller, "Fehlerabschätzungen für nichtsymmetrische Gauss-Quadraturformeln," Numer. Math. (To appear.)

3. H. BRASS, Quadraturverfahren, Vandenhoeck and Ruprecht, Göttingen, Zürich, 1977.

4. P. J. Davis \& P. Rabinowitz, Methods of Numerical Integration, Academic Press, New York, 1975.

5. W. Gautschi, “On Padé approximants associated with Hamburger series,” Calcolo, v. 20, 1983, pp. $111-127$.

6. W. GAUTSCHI \& R. S. VARGA, "Error bounds for Gaussian quadrature of analytic functions," SIAM J. Numer. Anal., v. 20, 1983, pp. 1170-1186.

7. W. Grobner \& N. Hofreiter (editors), Integraltafel, II Teil, Springer-Verlag, Wien, 1961.

8. G. HÄMMERLIN, "Fehlerabschätzungen bei numerischer Integration nach Gauss," Methoden und Verfahren der mathematischen Physik, Vol. 6 (B. Brosowski and E. Martensen, eds.), Bibliographisches Institut, Mannheim, Wien, Zürich, 1972, pp. 153-163.

9. R. Kumar, “A class of quadrature formulas,” Math. Comp., v. 28, 1974, pp. 769-778.

10. R. Kumar, “Certain Gaussian quadratures," J. Inst. Math. Appl., v. 14, 1974, pp. 175-182.

11. T. J. Rivin, The Chebyshev Polynomials, Wiley, New York, 1974. 